\title{
The Contribution of Radiotelegraphy to Geophysics
}

$\mathrm{O}^{\mathrm{N}}$ Thursday, April 27, the Kelvin lecture was delivered before the Institution of Electrical Engineers by Sir Frank Smith, who took as his subject, "The Travel of Wireless Waves". In the course of it, an interesting picture was presented of the position attained by modern research, both theoretical and experimental, on the mode of propagation of the electric waves employed in radio communication. Particular reference was made to the bearing of the results of this work on the general problems encountered in geophysics, and the manner in which modern research has provided the radio-geophysicist with an invaluable tool with which to probe into the higher reaches of the atmosphere.

The appropriateness of this lecture was expressed in the observation that Lord Kelvin first studied the oscillatory discharge of a condenser, and read a remarkable paper on it exactly eighty years ago. Kelvin also introduced a tool known as the 'image method', applicable to many problems of physics; and when this is applied to Hertz's dipole oscillator, the familiar pattern is obtained of the electric field due to an oscillating charge over a perfectly conducting plane. The energy radiated from such an oscillating dipole is in the form of electromagnetic waves, the properties of which are identical with light waves, due allowance being made for the difference in wave-length. The waves will travel in straight lines except for such deviation as will occur due to refraction or diffraction effects.

When Marconi successfully demonstrated the transmission of wireless signals around considerable portions of the earth's surface, much attention was directed to the problem of the manner in which the necessary deviation was produced. Fleming showed that refraction due to the variation of atmospheric density with altitude would produce a negligible effect, while similar conclusions were reached in a study of the diffraction effects, even making allowance for the conductivity of the earth and the resulting currents set up by the waves passing over it. Moreover, G. N. Watson found that in the case of diffraction the factor showing the dependence of the effect upon wavelength was of a different power from that obtained empirically and embodied in the well-known Austin-Cohen formula. The experimental investigations also produced later some remarkable results, such as the striking differences between day and night transmissions, and the unexpected potentialities of the shorter wave-lengths for long distance communication, in spite of their wellknown rapid absorption by the earth.

The need for a 'deflecting' region in the upper atmosphere was recognised in a note published by Prof. A. E. Kennelly in March 1902. A similar suggestion was made by Heaviside in an article written in June of the same year and afterwards published in the "Encyclopædia Britannica". In 1912, W. H. Eccles directed attention to the possible influence of the ionisation of the upper atmosphere on the propagation of electromagnetic waves through it, and to the resulting differences in day and night transmission. A few years later, Watson overcame his previous difficulty in a calculation of the field intensity of a wave propagated in the space between the earth and a conducting shell concentric with it.

In such circumstances it is natural to find the radio worker turning to the geophysicist for some knowledge of the properties of the atmosphere. By the aid of direct measuring instruments, the geophysicist has learnt much of the detailed physics of the troposphere and lower portions of the stratosphere; but it is not until a height of about $50 \mathrm{~km}$. is reached that much information of direct interest to the radiotelegraphist is supplied. At this height ozone is found as the result of the dissociation of oxygen by ultra-violet light, and this action is accompanied by ionisation, which naturally suffers a rapid reduction after sunset as a result of recombination. Further information on such ionisation in the higher regions of the atmosphere has been obtained from the study of aurora and also from investigations in terrestrial magnetism; and attention may be directed to the fact that Balfour Stewart in 1878 was the first to suggest that the daily changes in intensity of the earth's magnetic field are due to electric currents in the conducting portions of the upper atmosphere.

As a result of all this work, the radio investigator is informed that there exists, concentric with the earth, a spherical conducting layer, that the conductivity is due to electrons or ions, and that the conductivity varies with the direction so that electric waves penetrating the layer will be polarised. The probable ionising agents are ultraviolet light and charged or neutral particles projected from the sun, so that the layer is likely to be much more highly conducting on the side of the earth exposed to the sun than on the side in darkness. On this geophysicist's picture of the ionosphere no accurate scale of heights can be placed, nor can values of the ionisation density be assigned. It is merely known that the ozone is most dense at about $40 \mathrm{~km}$., and that the electron streams produce aurora at heights of from $90 \mathrm{~km}$. to above $500 \mathrm{~km}$.

With all this information at his disposal, the radiotelegraphist has been able to obtain at least a qualitative explanation of many of the phenomena encountered in his experimental investigations of the results of emitting waves from a radio station. In the first place, it is clear that, so far as the waves sent along the ground are concerned, the distance to which they travel before their field intensity falls to some specified value increases as the wave-length is increased ; and for this reason moderately long waves of wave-length 200 to 2,000 metres are employed for general broadcasting services. Next, the portion of the waves which are sent into the upper atmosphere 
will be refracted on entering the ionised portions and, upon reaching a height at which the density of ionisation is sufficiently great, the deviation will be such as to return the waves to the earth's surface. It has been found that on a wave-length of about 50 metres and using an antenna power of 5 kilowatts, these waves returning from the ionosphere reach the earth and affect a receiver at a minimum distance of the order of 2,500 miles. Since under the same conditions the ground wave will only be detectable at distances up to about 90 miles, there will be an annular space around the emitting station having inner and outer radii of 90 miles and 2,500 miles respectively in which no signals will be detectable. This is commonly known in radio engineering circles as the 'skip' effect and the larger radius above is termed the 'skipped distance'. As the wave-length is increased, this distance decreases steadily owing to the greater refraction effects taking place in the upper atmosphere; at the same time the range of the ground wave increases, and finally the two overlap for wave-lengths approximately in the shorter broadcasting band of 200 metres to 500 metres. Under these conditions, both ground and atmospheric waves reach the receiving station, and it is the interference effects resulting from these two sets of waves which cause the well-known phenomena of fading in broadcast reception, and of the variation of bearing in radio direction-finding.

In the later portion of his lecture, Sir Frank Smith described how the radio investigator has recently directed his attention to obtaining more conclusive proofs of the existence of the ionosphere and to the quantitative measurement of its properties; that is, to obtain information which the geophysicist has been unable to supply. Methods employed in other branches of physics have been adapted to radio communication for this purpose. One of these makes use of the interference effects obtained between the ground and atmospheric waves to determine the height of the ionosphere. The same objective has also been attained by using an echo method to measure the time taken by a pulse of electric waves to travel up to the ionised deflecting layer and return to earth. Owing to the very high velocity of the waves, this time interval is short-about one-thousandth of a second-and the cathode ray oscillograph has proved an invaluable tool in the determination of this quantity.

Working in this manner, the radiotelegraphist has conclusively proved the existence of the Kennelly-Heaviside layer in the ionosphere at an average height of about $100 \mathrm{~km}$. The measurements showed that for most nights the height of this ionised layer gradually increases, a maximum value being reached about one hour before sunrise. Further investigation showed that on certain occasions and with wave-lengths less than 400 metres, the height of the deflecting layer suddenly changes from $100 \mathrm{~km}$. to about $230 \mathrm{~km}$. It then became evident that on such occasions the density of ionisation in the Kennelly-Heaviside layer is insufficient for the deflection of the shorter waves, which thus penetrated this layer, but are ultimately deflected by a second layer above the first, the existence of which had not previously been suspected. This upper layer has been named after Prof. E. V. Appleton, who, with the co-operation of the Radio Research Board, has played the leading part in Great Britain in the investigations just described.

Having fixed the heights of these two important regions of the atmosphere, it has next been found possible to determine the density of the ionisation in these regions by ascertaining the limiting wavelength or frequency of the waves which will just penetrate the layer. Experiments carried out in this manner have shown that the ionisation is at its maximum about noon and decreases steadily as sunset is approached. After sunset, a rapid fall in ionisation takes place until, at about two hours afterwards, its value is only about onetenth of the maximum, and this then remains reasonably constant until shortly before sunrise. Two further facts which emerge from the work are that there is very little ionisation between the Kennelly-Heaviside and the Appleton layers, and that, for wave-lengths less than about 8 metres, the waves penetrate both layers and there is as yet no evidence of their return to earth from the upper atmosphere. As to the cause of the ionisation, some data obtained during the solar eclipse of August 31, 1932 appear to establish the fact that the principal ionising agent for the two layers is ultra-violet light. The possible bombardment of the earth by neutral particles is not yet wholly excluded, but undoubtedly the evidence so far is against it.

The review provided in this lecture shows very clearly that the modern radio research worker is obtaining detailed quantitative knowledge of certain portions of the atmosphere in a manner which was beyond the reach of the former geophysical methods of attack.

\section{Hydrogenated Motor Lubricants}

$\mathrm{T}$ HE recent display of advertisements has directed public attention to a new oil, "Essolube", a product representing the muchdiscussed process of hydrogenation successfully applied to the refining of petroleum. This particular lubricating oil is the product of many years research, involving much expenditure and the laying down of large-scale plant. The entry of hydrogenation as an established commercial process in the oil industry is an event of first-class technical and economic importance, and in many respects it will be a surprise to most people that the first satisfactory synthetic product in this field is a lubricant, a class of material distinguished for 